\title{
Underlying Event studies and Charged particle multiplicities in inelastic pp events with the ATLAS detector
}

\author{
Prafulla Kumar Behera \\ University of Iowa, 203 Van Allen Hall, \\ Iowa City, IA 52242-1479, United States of America \\ On behalf of the ATLAS Collaboration
}

\begin{abstract}
The measurement of the Underlying Event properties and charged particle multiplicities with the ATLAS detector in inelastic $p-p$ collisions at center of mass energy of $900 \mathrm{GeV}$ and $7 \mathrm{TeV}$ is presented. The charged particles density, its dependence on pseudo-rapidity and transverse momentum and its correlation with the average transverse momentum is measured for events with at least two charged particles in the kinematic range $|\eta|<2.5$ and $p_{T}>100$ $\mathrm{MeV}$. For Underlying Event studies, the transverse momentum of charged tracks is required to be greater than 500 $\mathrm{MeV}$. The measurements are compared with different Monte Carlo model predictions.
\end{abstract}

Keywords: soft QCD, multiparticle production

\section{Introduction}

The spectrum of charged particles created in proton-proton collisions is an important observable, that provides information about the production mechanism as well as the energy density. In order to perform precise physics measurements or search for new physics phenomena at hadron colliders, it is essential to have a good understanding not only of the Quantum chromodynamics (QCD) hard scattering process but also of the accompanying interactions with rest of the proton called "underlying event"(UE). Unlike hard interactions, interaction with low transverse momentum transfer from initial to final state (usually labeled as "soft") cannot be reliably calculated from perturbative QCD. The main technique to evaluate the size of their contribution to hard interaction hence relies on comparisons between the data and predictions from different phenomenological models, usually implemented in Monte Carlo (MC) event generators.

The first $p-p$ collisions at $\sqrt{s}=900 \mathrm{GeV}$ were delivered by the LHC and recorded by the ATLAS experiment [1] on the $23^{\text {rd }}$ of November 2009. On the $30^{\text {th }}$ of March 2010, the first collisions at $\sqrt{s}=7 \mathrm{TeV}$ were recorded. This paper discusses the latest charged particle multiplicity measurements done by ATLAS using about $7 \mu \mathrm{b}^{-1}$ of data at $\sqrt{s}=900 \mathrm{GeV}$ and about $190 \mu \mathrm{b}^{-1}$ data at $\sqrt{s}=7 \mathrm{TeV}$ [2]. The analysed samples contain a total of 10 million selected events with 210 million reconstructed tracks at $7 \mathrm{TeV}$ and a total of 360000 selected events with 4,5 million selected tracks at $900 \mathrm{GeV}$. The measurements are corrected for detector effects, in order to be comparable at hadron level. The following distributions are measured:

$$
\frac{1}{N_{e v}} \frac{d N_{e v}}{d n_{c h}}, \quad \frac{1}{N_{e v}} \frac{d N_{c h}}{d \eta}, \quad \frac{1}{N_{e v}} \frac{1}{2 \pi p_{T}} \frac{d^{2} N_{c h}}{d \eta d p_{T}}, \quad\left\langle p_{T}\right\rangle \text { vs. } n_{c h}
$$


where $N_{e v}$ is the number of events with at least two charged particles inside the selected kinematic range $\left(p_{T}>100\right.$ $\mathrm{MeV},|\eta|<2.5), N_{c h}$ is the total number of charged particles in the data sample, $n_{c h}$ is the number of charged particles in a given event, and $\left\langle p_{T}\right\rangle$ is the average charged particle $p_{T}$ for events with a given number of charged particles $n_{c h}$. Similar measurements were previously done by ATLAS [3, 4] for events with at least one charged particle with $p_{T}>500 \mathrm{MeV}$ and $|\eta|<2.5$. Results were also presented in a diffractive limited phase space, using events with at least six charged particles; a new minimum bias PYTHIA MC tune, AMBT1, was produced using those results [5].

The track with highest $p_{T}$ in the event (referred to as the "leading" track) is taken as representative of the alignment of the energy flow from the hardest scattering process. This direction is then used to isolate regions of $\eta-\phi$ space that are sensitive to different aspects of the UE. The azimuthal angular difference between charged tracks and the leading track is given by the angular magnitude $|\Delta \phi|=\left|\phi-\phi_{\text {leadingtrack }}\right|$ and three distinct azimuthal regions are defined as follows: $|\Delta \phi|<60^{\circ}$ is the "towards region", $60^{\circ}<|\Delta \phi|<120^{\circ}$ is the "transverse region" and $|\Delta \phi|>120^{\circ}$ is the "away region". The measured distributions for UE studies are charged particle multiplicity, charged particle scalar $P_{T}$ sum, charged particle mean $p_{T}$ and angular distribution. The same event, track selection (except $p_{T}>500 \mathrm{MeV}$ ) and correction procedure are used for both studies.

\section{Analysis overview}

\subsection{Event and track selection:}

Events are selected based on a single-arm trigger which requires at least one hit on either of the two Minimum Bias Trigger Scintillators disks situated at $2.12<|\eta|<3.8$. In this measurement a single-arm trigger was chosen instead of a double-arm coincidence trigger, in order to provide a more inclusive measurement of inelastic collisions, and therefore minimize model-dependent corrections applied to account for residual diffractive components in a measurement of non-single-diffractive events.

Furthermore, a reconstructed primary vertex is required to be present in the event. This helps to reduce background events and provides a geometrical reference where particles from the primary interaction are supposed to originate from, thereby helping to reduce the contamination from secondary and fake tracks. The tracks that are used to reconstruct the primary vertex are selected within the same phase space as the tracks used for the analysis. Due to the fact that we need at least two tracks to reconstruct a primary vertex, events with less than two tracks are discarded.

Tracks used for the analysis are selected within the analysis phase space in such a way to reject secondary tracks that originate from long lived neutral hadrons $\left(K_{s}^{0}\right.$ and $\left.\Lambda^{0}\right)$ or from hadronic interactions of particles with the detector material, and to reject poorly measured tracks that might introduce biases in the measured distributions. Selection criteria involve impact parameter ${ }^{1}$ requirements, minimal number of hits requirements and a reconstruction $\chi^{2}$ requirement. In case of events with more than one vertex, the events with four or more tracks associated to the second vertex are rejected.

\subsection{Hadron level corrections:}

All distributions are corrected back to the hadron level of inelastic proton-proton collisions removing detector effects. At the event level, a correction is applied to account for trigger and vertex efficiencies; a weight given by the following expression is assigned to each event as $w_{e v}\left(n_{\text {sel }}^{B S}\right)=\frac{1}{\epsilon_{\text {trig }}\left(n_{\text {sel }}^{B S}\right)} \cdot \frac{1}{\epsilon_{v t x}\left(n_{\text {sel }}^{B S}\right)}$ where $\epsilon_{t r i g}\left(n_{\text {sel }}^{B S}\right)$ and $\epsilon_{v t x}\left(n_{\text {sel }}^{B S}\right)$ are respectively the trigger and vertex efficiencies. Both efficiencies are measured on data and parametrized as a function of the number of selected tracks. In this procedure, the tracks are extrapolated to the nominal collision point (the beam spot) when applying the impact parameter requirements.

In addition to the event level correction, a track level correction is applied to account for tracking efficiency $\left(\epsilon_{t r k}\left(p_{T}, \eta\right)\right)$, the rate of secondary and fake tracks $\left(f_{\text {sec }}\left(p_{T}, \eta\right)\right)$ and the rate of tracks passing track selection that originate from the region outside the analysis kinematic range $\left(f_{O K R}\left(p_{T}, \eta\right)\right)$. Each track is assigned a weight given by:

$$
w_{t r k}\left(n_{s e l}^{B S}, p_{T}, \eta\right)=w_{e v}\left(n_{s e l}^{B S}\right) \cdot \frac{1}{\epsilon_{t r k}\left(p_{T}, \eta\right)} \cdot\left(1-f_{s e c}\left(p_{T}, \eta\right)-f_{O K R}\left(p_{T}, \eta\right)\right)
$$

\footnotetext{
${ }^{1}$ The impact parameter is defined as the distance of closest approach of the track to the primary vertex. It is computed in both the transverse plane $d_{0}$ and along the longitudinal direction $z_{0}$.
} 

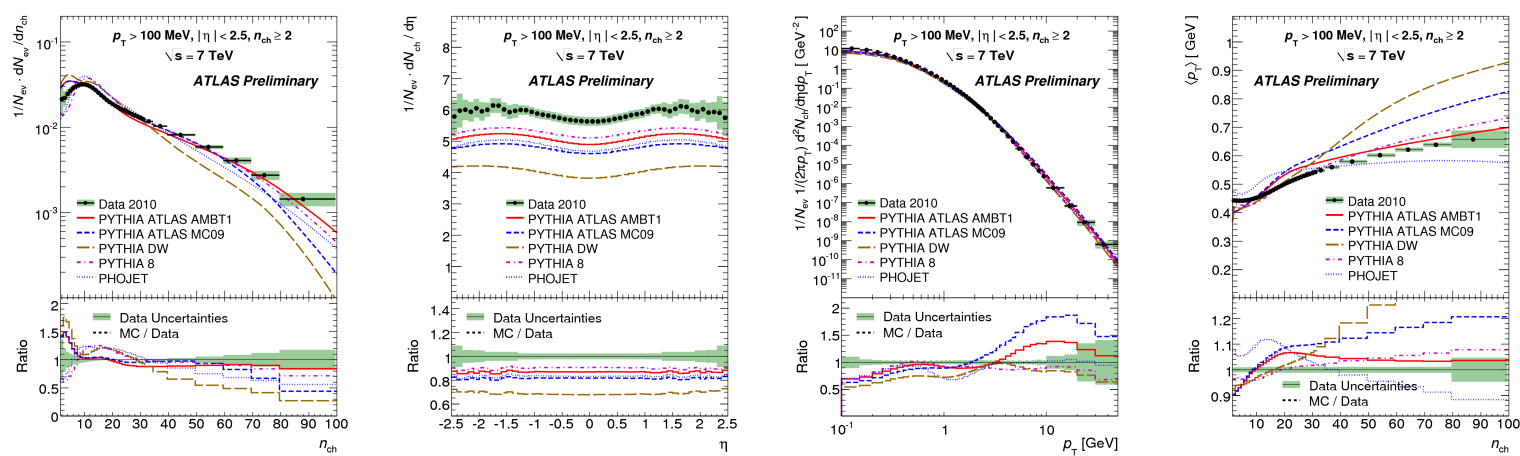

Figure 1: Charged-particle multiplicities for events with $n_{c h} \geq 2$ within $p_{T}>100 \mathrm{MeV}$ and $|\eta|<2.5$ at $\sqrt{s}=7 \mathrm{TeV}$. The panels show the charged particle multiplicity (a), the charged particle multiplicity as a function of the pseudo-rapidity (b) and transverse momentum (c), and the average transverse momentum as a function of the number of charged particles in the event (d). The data are compared to various particle level MC predictions.

The tracking efficiency is estimated for MC events and is parametrized as a function of $p_{T}$ and $\eta$; the main systematic uncertainty assigned to the tracking efficiency is due to the modeling of the interaction of charged particles with the material of the detector and to the estimated amount of material present in the detector. The uncertainty on tracking efficiency was estimated from data by comparing to MC the bias on the reconstructed $K^{0}$ mass and the fraction of tracks in the pixel detector that are matched to a track in the full inner detector.

A final correction is applied to correct the selected track multiplicity, $n_{\text {sel }}$, to the charged particle multiplicity, $n_{c h}$. The correction implements an iterative bayesian unfolding algorithm. According to Bayes theorem, the charged particle multiplicity probability distribution $P\left(n_{c h}\right)$ is given as a function of the selected track multiplicity probability distribution $P\left(n_{\text {sel }}\right)$ by:

$$
P\left(n_{c h}\right)=\sum_{n_{s e l}} P\left(n_{s e l}\right) \cdot P\left(n_{c h} \mid n_{s e l}\right), \quad P\left(n_{c h} \mid n_{s e l}\right)=\frac{P\left(n_{s e l} \mid n_{c h}\right) \cdot P\left(n_{c h}\right)}{P\left(n_{s e l}\right)}
$$

where $P\left(n_{c h} \mid n_{s e l}\right)$ is the probability that an event contains $n_{c h}$ particles when it is observed with $n_{\text {sel }}$ tracks. This probability can be evaluated for MC events but would depend on the simulated distribution (the prior). This can be overcome by an iterative procedure where at each iteration, $\mathrm{MC}$ events are reweighted according to the corrected distribution observed in data in the previous iteration; the procedure converges after a few iterations. The main systematic uncertainties that affect this unfolding procedure are due to systematic uncertainty on the tracking efficiency, and to the fact that the MC events were simulated with a different $p_{T}$ spectrum than the one observed from data. Both effects propagate to the migration probabilities $P\left(n_{c h} \mid n_{s e l}\right)$ which are measured for MC. A similar approach is applied to correct for non-Gaussian resolution effects in the $p_{T}$ distribution.

\section{Minimum bias, UE measurements and Conclusion}

The charged particle multiplicity distributions defined in Eq. 1 are shown for $7 \mathrm{TeV}$ data in figure 1 and compared to various model predictions. The lack of understanding of diffractive physics seems to be reflected in the data MC comparison at low $n_{c h}$ and high $p_{T}$ : the predictions from various models differ from each other and do not agree well with the data. Large discrepancies between data and model predictions are also observed at high $n_{c h}$ and high $p_{T}$. The AMBT1 tune which was tuned using ATLAS data at $7 \mathrm{TeV}$, seems to agree better with data. The ATLAS experiment measures on average $5.635 \pm 0.002$ (stat.) \pm 0.149 (syst.) charged particles with $p_{T}>100 \mathrm{MeV}$ per unit of pseudo-rapidity at $\eta=0$ at $\sqrt{s}=7 \mathrm{TeV}$, and $3.486 \pm 0.008$ (stat. $) \pm 0.077$ (syst.) at $\sqrt{s}=0.9 \mathrm{TeV}$.

The measured distributions for UE are charged particle multiplicity, charged particle scalar $P_{T}$ sum, charged particle mean $p_{T}$ and angular distribution, shown in figure 2(a), 2(b), 2(c), and 2(d) respectively. These measurements show a significant differences between MC models and the measured distributions. Most of the tunes predict less 
activity in the transverse region than is actually observed. The charged particle multiplicity in the plateau of the transverse region distribution was found to be about two times higher than that of minimum bias particle density in the overall event.
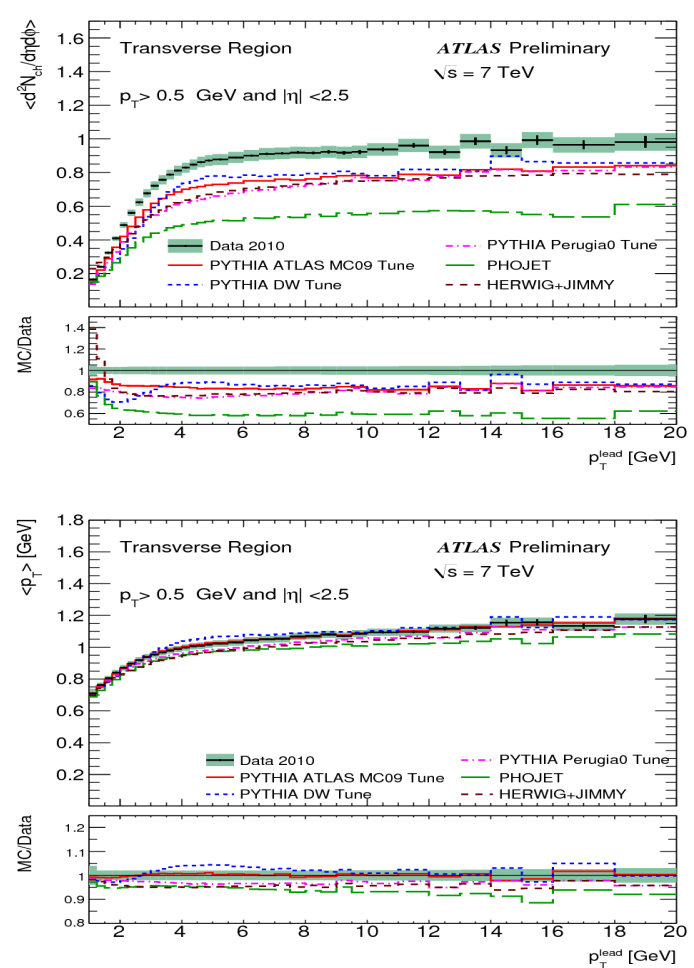
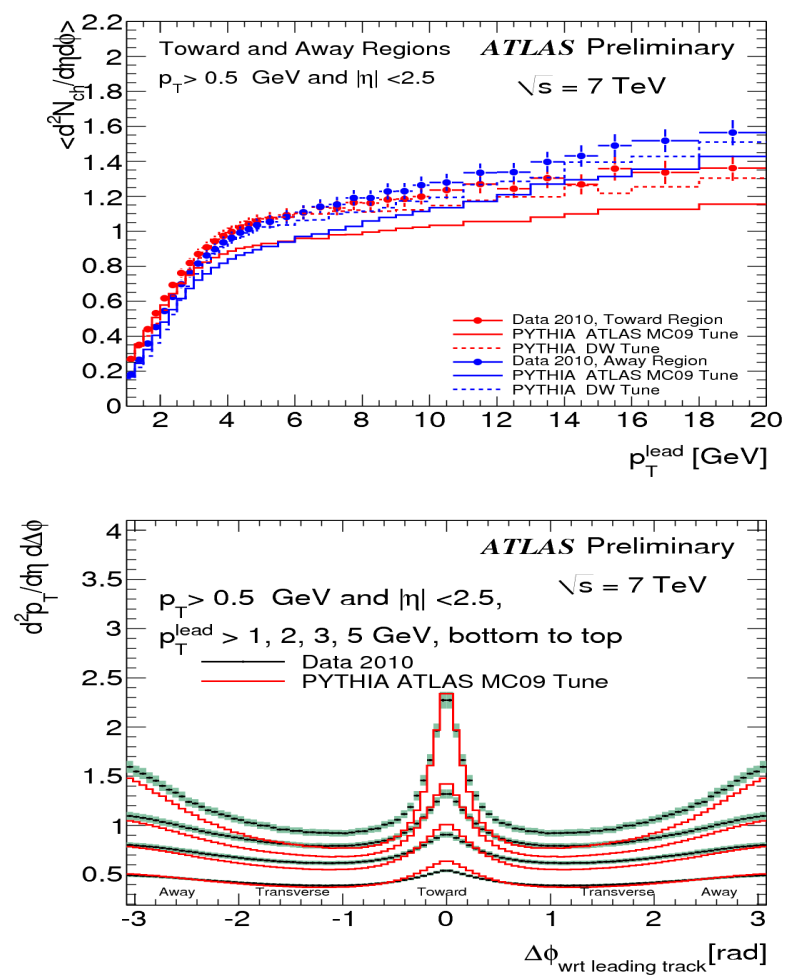

Figure 2: Charged-particle multiplicities with $p_{T}>500 \mathrm{MeV}$ and $|\eta|<2.5$ at $\sqrt{s}=7 \mathrm{TeV}$ [6]. Top Left:The panels show the density of charged particles as a function of the leading track $p_{T}$ (a), Top Right:the scalar sum $p_{T}$ density of charged particles as a function of the leading track $p_{T}$ (b) and Bottom Left: the mean $p_{T}$ of charged particles as a function of the leading track $p_{T}$ (c), and Bottom Right: the phi distribution of track $p_{T}$ densities with respect to the leading track rotated to $\phi_{\text {leading }}=0$, excluding the leading track (d). The data are compared to various particle level MC predictions.

\section{References}

[1] The ATLAS Collaboration, The ATLAS Experiment at the CERN Large Hadron Collider, JINST 3, S08003, 2008.

[2] The ATLAS Collaboration, Charged particle multiplicities in pp interactions for track $p_{T}>100 \mathrm{MeV}$ at $\sqrt{\mathrm{s}}=0.9$ and 7 TeV measured with the ATLAS detector at the LHC , ATLAS-CONF-2010-046. http://cdsweb.cern.ch/record/1281296

[3] The ATLAS Collaboration, Charged-particle multiplicities in pp interactions at $\sqrt{s}=900$ GeV measured with the ATLAS detector at the LHC, arXiv:1003.3124v1 [hep-ex].

[4] The ATLAS Collaboration, Charged particle multiplicities in pp interactions at $\sqrt{s}=7$ TeV measured with the ATLAS detector at the LHC, ATLAS-CONF-2010-024. http://cdsweb.cern.ch/record/1277656

[5] The ATLAS Collaboration, Charged particle multiplicities in pp interactions at $\sqrt{s}=0.9$ and 7 TeV in a diffractive limited phase space measured with the ATLAS detector at the LHC and a new PyTHIA6 tune, ATLAS-CONF-2010-031. http://cdsweb.cern.ch/record/1277665

[6] The ATLAS Collaboration, Track-based underlying event measurements in pp collisions at $\sqrt{s}=900 \mathrm{GeV}$ and $7 \mathrm{TeV}$ with the ATLAS Detector at the LHC, ATLAS-CONF-2010-081. http://cdsweb.cern.ch/record/1298845 